Journal of Animal and Veterinary Advances 18 (3): 71-77, 2019

ISSN: $1680-5593$

(C) Medwell Journals, 2019

\title{
Comparison of the Efficiency of Landmark-Based and Semilandmark-Based Geometric Morphometrics to Diagnose Three Anopheles Species
}

\author{
${ }^{1}$ Tanawat Chaiphongpachara and ${ }^{2}$ Sedthapong Laojun \\ ${ }^{1}$ College of Allied Health Science, Suan Sunandha Rajabhat University, Bangkok, Thailand \\ ${ }^{2}$ Bachelor of Public Health, College of Allied Health Sciences, \\ Suan Sunandha Rajabhat University, 75000 Samut Songkhram, Thailand
}

\begin{abstract}
In this study, our objective was comparison of the efficiency of Geometric Morphometrics (GM) to diagnose three Anopheles species including males and females of An. barbirostris s.1. van der Wulp An. epiroticus Linton and Harbach and An. subpictus s.1. Grassi in Thailand by using the Land Mark-based (LM), semil and mark-based (LMSL) methods of GM. The results of the size and shape analysis revealed clear similarities of both the LM and LMSL methods. The analysis of wing size based on centroid size in both the LM and LMSL methods indicated that the wing size patterns were different in all species except between $A n$. epiroticus and An. subpictus s.1. females. All pairwise comparisons of wing shape based on Mahalanobis distance scores presented significant differences (permutation test: 1,000 cycles, $\mathrm{p}<0.05$ ). Cross-validated reclassification scores based on assessing the accuracy of cluster recognition showed that the LMSL method was slightly more efficient than the LM method ( 96 vs. 91\%). This study revealed that using the LMSL method can better separate the females and males of An. barbirostris s.1., An. subpictus s.1. and An. epiroticus than LM which can be useful in guiding the identification of correct vectors for malaria control.
\end{abstract}

Key words: Geometric morphometric, landmark and semi-landmark-based approach, Anopheles barbirostris s.1. van der Wulp, Anopheles epiroticus Linton and Harbach, Anopheles subpictus s.1. Grassi, malaria

\section{INTRODUCTION}

Malaria is a life-threatening disease that can present worldwide and which is caused by Plasmodium parasites transmitted by female Anopheles mosquitoes (Service, 2008). WHO. (2017) reported that there were 19 million cases and 435,000 deaths worldwide as a result of malaria. Generally, this disease occurs in the tropical and subtropical zones that are associated with Anopheles vectors (WHO., 2013). Anopheles mosquitoes belong to the subfamily Anophelinae which includes 488 formally recognized species (Tainchum et al., 2015). Importantly, not all species of Anopheles mosquito are able to transmit malaria. Anopheles vectors and non-vectors are related in terms of vectorial capacity and the ability to transmit Plasmodium pathogens to humans (Brady et al., 2016). The correct species identification of mosquito vectors is important for the selection of appropriate control methods. However, species identification based on morphological characteristics is quite difficult in some species with very similar morphology (WHO., 2007) or in the case of samples that have been damaged in the field (Chaiphongpachara et al., 2018). Molecular techniques are one of the methods available to help solve this problem but they are expensive and require sophisticated equipment to perform.

Geometric Morphometrics (GM) is a popular alternative approach used in entomological studies, especially in the species identification of mosquito vectors (Wilke et al., 2016). Recently, GM has been used to identify three member species of Anopheles in the Maculatus group in Thailand including An. maculatus An. sawadwongporni and An. pseudowillmori (Chaiphongpachara et al., 2019). In addition, GM has been applied to An. barbirostris s.1. and An. subpictus s.l. in the Ratchaburi province of Thailand Chaiphongpachara et al., 2018). In both cases, GM was highly effective in identifying Anopheles mosquitoes. The Land Mark-based method (LM) is one of GM technique and is widely used, offering the advantages of being easy to use, speedy analysis and cheap cost (Dujardin, 2008). The coordinates of anatomical landmarks are typically used in LM analysis which often defines these landmarks

Corresponding Author: Tanawat Chaiphongpachara, College of Allied Health Sciences, Suan Sunandha Rajabhat University, 75000 Samut Songkhram, Thailand 
spread across the wings of mosquitoes. Type 3 landmarks or semi landmarks (also called sliding semi landmarks) is a type of landmark that has been developed for the description of curved lines (Bookstein, 1997). Previous research has reported that landmarks combined with semilandmarks (also called Land Mark-and Semi-Landmark based method, LMSL) is more effective in identifying many species of tsetse flies than LM (Kaba et al., 2017).

In this study, our objective was comparison of the efficiency of both the LM and LMSL GM methods to diagnose three Anopheles species including males and females of $A n$. barbirostris s.1. van der Wulp, An. epiroticus Linton and Harbach and An. subpictus s.l. Grassi in Thailand. These three species of Anopheles mosquitoes are important vectors in malaria transmission in Southeast Asia. An. barbirostris s.1. is recognized as a significant malaria vector and has been found to be associated with infection with the protozoan parasite Plasmodium in many areas of Thailand (Rattanarithikul et al., 1996). An. epiroticus is a secondary vector of malaria in Thailand found near coastal zones and associated with brackish water habitats (Sumruayphol et al., 2010; Chaiphongpachara and Sumruayphol, 2017). To our knowledge, there is no report showing the relevance between An. subpictus s.l. and malaria in Thailand but for other countries such as Indonesia, India and Sri Lanka it is a major problem.

\section{MATERIALS AND METHODS}

Anopheles mosquito collection: Female and male adult Anopheles mosquitoes were captured using a Mosquito Magnet Independence Trap (Woodstream Corporation, Lititz, PA, USA) in two provinces in Thailand during June to August 2015. An. barbirostris s.1., An. epiroticus and An. subpictus s.l. were used in this study. An. barbirostris s.1. and An. subpictus s.1. were collected at border areas in Ratchaburi province $\left(13^{\circ} 22^{\prime} 36.0^{\prime \prime} \mathrm{N}, 99^{\circ} 16^{\prime} 34.9^{\prime \prime} \mathrm{E}\right)$ while, An. epiroticus was collected at coastal areas in Samut Songkhram Province (1324'32.52'N, $\left.100^{\circ} 0^{\prime} 41.40^{\prime \prime} \mathrm{E}\right)$. All mosquito collections were conducted from 18:00 until 06:00 and were performed once a week. Anopheles mosquitoes were sent to the laboratory of the College of Allied Health Sciences, Suan Sunandha Rajabhat University, Samut Songkhram Provincial Education Center to identify species the following morning using the morphological keys (Rattanarithikul et al., 2010).

Slide preparation: For GM analysis, the number of samples should be twice the number of landmarks. Therefore, 30 mosquitoes per species and sex were randomized and used for LM and LMSL analysis.

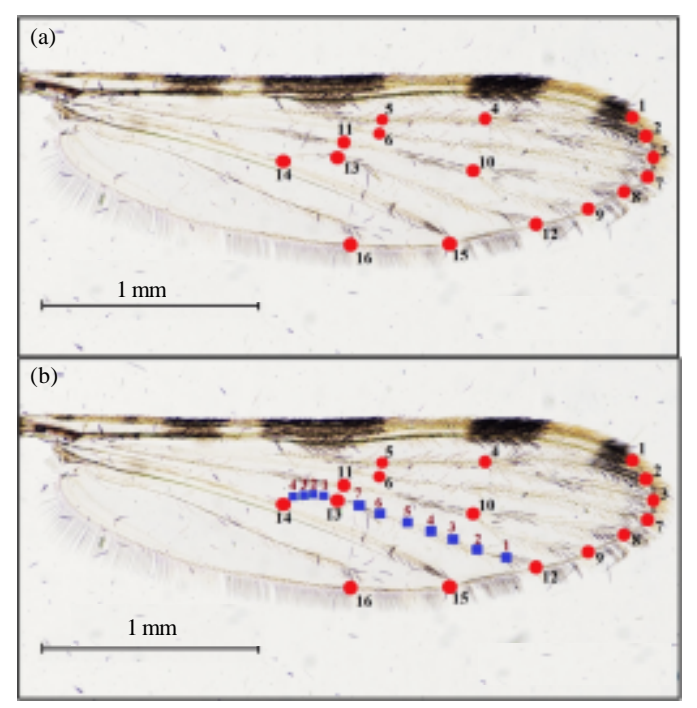

Fig. 1: Coordinates of landmarks and semilandmarks on mosquito wings for GM analysis: a) LM analysis and b) LMSL analysis

Complete left wings were removed from the collected mosquito bodies and mounted on microscope slides under a coverslip using Hoyer's solution as a permanent mounting medium. A Nikon DS-Ril SIGHT digital camera coupled to a Nikon SMZ745T stereomicroscope (Nikon, Tokyo, Japan) with $40 \mathrm{x}$ magnification was used to photograph wing images.

Both the LM and LMSL methods used the same analysis process but differ in their manners of determining the points of coordinates shown in Fig. 1a, b. Coordinates of 16 landmarks were selected and digitized for the LM method (Fig. 1a). In the LMSL methodcoordinates of 16 landmarks (same Landmarks in LM) combined with 11 semil and marks which were divided into two curved lines of the wing vein including seven semilandmarks between landmarks 12 and 13 and four semilandmarks between landmarks 13 and 14 were digitized (Fig. 1b).

\section{Geometric morphometric analysis}

Repeatability test: A repeatability test involved the repeated evaluation of the accuracy or measurement error of landmarks. The measurement error in this study was examined using the "Repeatability (R)" index (Arnqvist and Martensson, 1998). Landmark repeatability was tested in 10 wings per species in each sex selected randomly which were digitized twice by the same person.

Size analysis: The Centroid Size (CS) isometric estimator derived from coordinates was used to analyze the overall wing size among female and male Anopheles mosquitoes. $\mathrm{CS}$ is defined as "the square root of the summation of the 
squared distances between the center of the configuration and each landmark individual" (Bookstein, 1991). Differences in wing CS in each Anopheles species were compared by a nonparametric method $(1,000$ cycles) with bonferroni correction at a risk level of $5 \%$.

Shape analysis: Wing shape variables were computed using the generalized Procrustes analysis superimposition algorithm and the subsequent projection of the Procrustes residuals into an Euclidean space (Rohlf, 1999) with the resulting variables being partial warps. Discriminant analys is as a statistical method was performed to explore shape dissimilarity between species. Results from this were presented as a factor map by Canonical Variate Analysis (CVA) and were used to calculate the Mahalanobis distance scores. Wing shape differences in each species based on Mahalanobis distances were computed using a nonparametric method (1,000 cycles) with Bonferroni correction at a risk level of 5\%.

Classification tests and morphological trees: A cross-validated reclassification test was used to assess the accuracy of cluster recognition based on Mahalanobis distance scores. For assessment, each mosquito wing was reclassified according to its wing shape similarity within each group. Morphological trees of both the LM and LMSL methods were established using the neighbor-joining method to investigate the nearness of wing shape in each species. Thirty male and female Aedes aegypti were used as an out-group in the morphological trees.

Software: The CLIC package as a GM software was used for LM and LMSL analysis in this study and is freely available at http://xyom-clic.eu/. The neighbor-joining trees were developed using the R Software ( $\mathrm{R}$ Foundation, Vienna, Austria) which is freely available at https://cran.r-project.org/.



\section{RESULTS AND DISCUSSION}

Repeatability: The comparison of two repeated sets of measurements for the same wing images showed good repeatability scores for wing size based on CS $(\mathrm{R}=0.98$ for males and 0.97 for females) and wing shape based on relative warps ( $\mathrm{R}=0.95$ for males and 0.94 for females ).

Wing size: The morphometric analysis of wing CS for both the LM and LMSL methods yielded the same results. The mosquito with the largest wings was the $A n$. barbirostris s.1. female and that with the smallest wings was the An. subpictus s.1. male. Wing CS variations between species and sexes of each Anopheles species in both the LM and LMSL methods are shown in Fig. 2a, b. Sexual size dimorphism was different among all species with the females generally being larger than the males. The mean wing CS values of female and male Anopheles mosquitoes were significantly different in all pairwise comparisons $(\mathrm{p}<0.05$, Table 1$)$.

Wing shape: The factor maps of the discriminant analysis generated from CVA (Fig. 3) for exploring shape dissimilarities between species and sexes revealed that almost all of the specimens could be separated into distinct groups, especially, when considering the factor map of LMSL. All pairwise comparisons of wing shape based on Mahalanobis distance scores presented significant differences (permutation test: 1,000 cycles, $\mathrm{p}<0.05$, Table 2).

Accuracy of cluster recognition and neighbor-joining trees: The percentages of correctly classified samples obtained from the cross-validation reclassification test revealed high scores for both methods, specifically, 91\% for the LM method and $96 \%$ for the LMSL method (Table 3). The investigations of nearness of wing shape in each species and sex were visualized by the neighbor

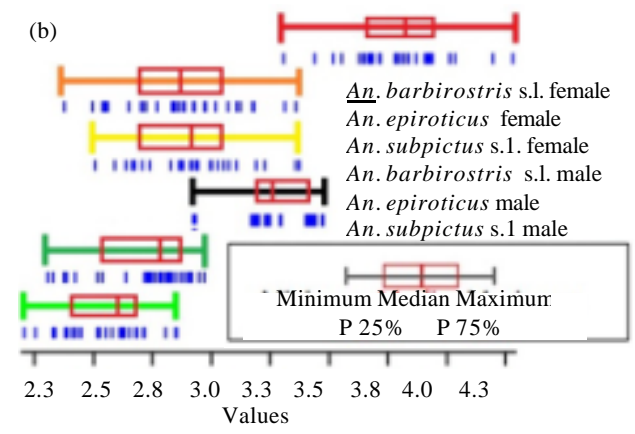

Fig. 2: Wing CS variation between species and sexes in both: a) LM and LMS b) methods (units are pixels converted to millimeters ( $\mathrm{mm}$ ), shown as quantile boxes. Each box shows the group median ( 25 th and 75 th percentiles) and the vertical bars under the boxes represent the wings 



Fig. 3: Discriminant analysis of both: a) LM and b) LMSL methods. The horizontal axis is the first discriminant factor and the vertical axis is the second discriminant factor

Table 1: Wing CS comparisons between species and sexes of Anopheles mosquitoes

\begin{tabular}{|c|c|c|}
\hline \multirow[b]{2}{*}{ Species } & \multicolumn{2}{|l|}{ Mean \pm SD (Min-Max) } \\
\hline & $\mathrm{LM}$ & LMSL \\
\hline An borbirostris s.l. female & $3.22 \pm 0.03(2.84-3.61)^{\mathrm{a}}$ & $3.84 \pm 0.04(3.36-4.30)$ \\
\hline An epiroticus female & $2.44 \pm 0.04(2.07-2.82)^{b}$ & $2.94 \pm 0.06(2.46-3.42)^{b}$ \\
\hline An. subpictus s.l. female & $2.45 \pm 0.03(2.14-2.83)^{b}$ & $2.95 \pm 0.04(2.56-3.39)$ \\
\hline An barbirostris s.l. male & $2.78 \pm 0.01(2.52-2.92)^{\mathrm{c}}$ & $3.32 \pm 0.02(2.99-3.50)^{\mathrm{c}}$ \\
\hline An epiroticus male & $2.29 \pm 0.03(1.97-2.50)^{\mathrm{d}}$ & $2.76 \pm 0.04(2.36-3.019)$ \\
\hline An subpictus s.l. male & $2.17 \pm 0.02(1.88-2.40)^{\mathrm{e}}$ & $2.61 \pm 0.03(2.26-2.89)^{e}$ \\
\hline
\end{tabular}

Table 2: Comparisons of Mahalanobis distance scores between species and sexes of Anopheles mosquitoes

\begin{tabular}{|c|c|c|c|c|c|c|}
\hline Methods/species & $\begin{array}{l}\text { An. barbirostris } \\
\text { male }\end{array}$ & $\begin{array}{l}\text { An. epiroticus } \\
\text { female }\end{array}$ & $\begin{array}{l}\text { An subpictus } \\
\text { female }\end{array}$ & $\begin{array}{l}\text { An. barbirostris } \\
\text { male }\end{array}$ & $\begin{array}{l}\text { Anepiroticus } \\
\text { male }\end{array}$ & $\begin{array}{l}\text { An subpictus } \\
\text { male }\end{array}$ \\
\hline \multicolumn{7}{|l|}{ LM } \\
\hline An barbirostris s.l. female & - & & & & & \\
\hline An epiroticus & $9.36^{*}$ & - & & & & \\
\hline An. subpictus s.l. female & $7.73 *$ & $5.05^{*}$ & - & & & \\
\hline An barbirastris s.l. male & $5.70^{*}$ & $12.33^{*}$ & $11.52 *$ & - & & \\
\hline An epiroticus & $9.55^{*}$ & $7.13^{*}$ & $9.32^{*}$ & $9.61^{*}$ & - & \\
\hline \multirow{2}{*}{\multicolumn{7}{|c|}{$\begin{array}{l}\text { An. subpictus s.1. Inlale } \\
\text { LMSL }\end{array}$}} \\
\hline & & & & & & \\
\hline An. barbirostris s.l. female & - & & & & & \\
\hline An epiroticus & $16.73^{*}$ & - & & & & \\
\hline An subpictus s.1. female & $13.29^{*}$ & $7.27^{*}$ & - & & & \\
\hline An barbirostris s.l. male & $6.53^{*}$ & $18.94 *$ & $16.39 *$ & - & & \\
\hline An epiroticus & $17.33^{*}$ & $9.49^{*}$ & $12.63^{*}$ & $17.17^{*}$ & - & \\
\hline An subpictus s.l. male & $12.80^{*}$ & $9.51^{*}$ & $9.77^{*}$ & $12.59^{*}$ & $7.61^{*}$ & - \\
\hline
\end{tabular}

LM $=$ Landmark-based Method, LMSL $=$ Semilandmark-based Method. ${ }^{*}$ indicates statistical significance at $p<0.05$

Table 3: Percentages of cross-validated reclassification scores based on wing shape similarities

\begin{tabular}{llc}
\hline & Accuracy of classification (assigned/observed) \\
\cline { 2 - 3 } Species & LM(\%) & LMSL(\%) \\
\hline An. barbirostris s.l. female & $100(30 / 30)$ & $100(30 / 30)$ \\
An. epiroticus female & $86(26 / 30)$ & $86(26 / 30)$ \\
An. subpictus s.1. female & $90(27 / 30)$ & $96(29 / 30)$ \\
An. barbirostris s.l. male & $100(30 / 30)$ & $100(30 / 30)$ \\
An. epiroticus male & $80(24 / 30)$ & $90(27 / 30)$ \\
An. subpictus s.1. male & $86(26 / 30)$ & $100(30 / 30)$ \\
Total & $91(163 / 180)$ & $96(172 / 180)$ \\
\%= Percentage, LM = Landmark-based Method, LMSL = Semilandmark- &
\end{tabular}

joining trees based on the Procrustes distances. Both the LM and LMSL methods led to the same morphological tree pattern which located $A n$. barbirostris s.1. females, An. epiroticus females, $A n$. subpictus s.1. females, An. epiroticus males and An. subpictus s.1. males into the same cluster but separated out An. barbirostris s.1. males (Fig. 4). Female and male Aedes aegypti were additionally clearly separated from all other Anopheles mosquitoes. 

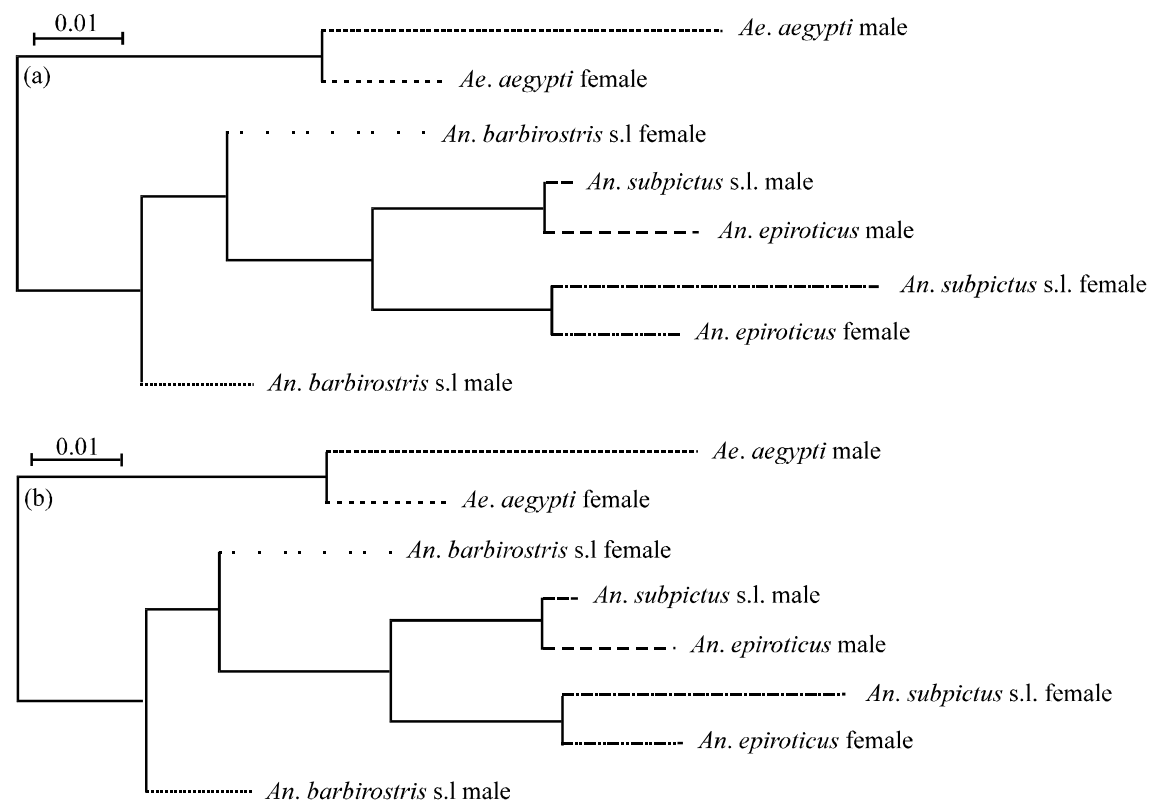

Fig. 4: Neighbor-joining trees of both: a) LM and b) LMSL GM method

The present research evaluated and compared the efficiency of GM between the LM and LMSL GM methods to diagnose three female and male Anopheles species in Thailand including An. barbirostris s.1., An. epiroticus and An. subpictus s.l. mosquitoes. The results of the size and shape analysis reveal clear similarities of both the LM and LMSL methods.

The analysis of wing size based on CS using both the LM and LMSL methods indicated wing size patterns were almost all different in all species except in the pairing of An. epiroticus and An. subpictus s.l. females. However, the consideration of wing size is not a good primary way for diagnosing species because it is not stable and is highly sensitive to the environment. The influence of the surrounding environment during the larval stage such as the quantity or quality of food and temperature affects adult mosquitoes directly (Jirakanjanakit et al., 2017). Most wing size analysis data are often used to study morphological variations in different environments, rather than being used for mosquito species identification (Lorenz et al., 2017). Previous research has been conducted on the influence of environmental heterogeneity on An. darlingi in Northwest Colombia and found that wing size differences may reflect plastic responses rather than genetic divergence due to low genetic differentiation and high gene flow in mosquito populations (Altamiranda-Saavedra et al., 2017).

The wing shape of mosquitoes has been recognized as an important evidence of microevolution and identity of each species separate from genetic expression
(Suesdek, 2018). Thus, wing shape is often used to diagnose species of mosquito vectors using by GM. The results of the wing shape evaluation of both GM methods in the present investigation indicated significant differences exist in wing shape in all species and sexes. These results were consistent with those of other recent research in Thailand that found that GM has the potential to separate An. barbirostris s.1. and An. subpictus s.1. from one another (Chaiphongpachara, 2018). Cross-validated reclassification scores based on assessing the accuracy of cluster recognition have shown that the LMSL method is slightly more efficient than the LM method (96 vs. 91\%). These analysis findings suggest that the use of landmarks in combination with the analysis of the important curved lines of the wing vein using the LMSL method can increase the efficiency of stratifying An. barbirostris s.l. An. subpictus s.l. and An. epiroticus females and males. This heightened efficiency for diagnosing species may be due to the increasing coordinates for analysis and the fact that the positioning of these curve lines constitutes a unique characteristic that helps to separate species from each other. This is consistent with previous research that found that LMSL has more efficiency on species identification than LM in many species of tsetse flies (Kaba et al., 2017).

\section{CONCLUSION}

The GM technique is a tool to help diagnose certain species of mosquitoes that are difficult to separate from 
one another using morphological characteristics alone. LM is one method of GM that is commonly used to identify mosquito vectors because of its ease and quickness of use as well as cheapness. This study revealed that using the LMSL can better separate $A n$. barbirostris s.1. An. subpictus s.1. and An. epiroticus females and males than LM. This information will useful for guiding the identification of correct vectors for malaria control.

\section{ACKNOWLEDGEMENT}

Researchers would like to thank the College of Allied Health Science, Suan Sunandha Rajabhat University, Thailand, for support of the research.

\section{REFERENCES}

Altamiranda-Saavedra, M., J.E. Conn and M.M. Correa, 2017. Genetic structure and phenotypic variation of Anopheles darlingi in Northwest Colombia. Inf. Gen. Evol., 56: 143-151.

Arnqvist, G. and T. Martensson, 1998. Measurement error in geometric morphometrics: Empirical strategies to assess and reduce its impact on measures of shape. Acta Zool. Academiae Scientiarum Hungaricae, 44: 73-96.

Bookstein, F.L., 1991. Morphometric Tools for Landmark Data: Geometry and Biology. Cambridge University Press, Cambridge, London.

Bookstein, F.L., 1997. Landmark methods for forms without landmarks: Morphometrics of group differences in outline shape. Med. Image Anal., 1: 225-243.

Brady, O.J., H.C.J. Godfray, A.J. Tatem, P.W. Gething and J.M. Cohen et al., 2016. Vectorial capacity and vector control: Reconsidering sensitivity to parameters for malaria elimination. Trans. Royal Soc. Trop. Med. Hyg., 110: 107-117.

Chaiphongpachara, T. and S. Sumruayphol, 2017. Species diversity and distribution of mosquito vectors in coastal habitats of Samut Songkhram province, Thailand. Trop. Biomed., 34: 524-532.

Chaiphongpachara, T., 2018. Comparison of landmark-and outline-based geometric morphometrics for discriminating mosquito vectors in Ratchaburi Province, Thailand. BioMed Res. Intl., 2018: 1-10.

Chaiphongpachara, T., P. Sriwichai, Y. Samung, J. Ruangsittichai and R.E.M. Vargas et al., 2019. Geometric morphometrics approach towards discrimination of three member species of maculatus group in Thailand. Acta Trop., 192: 66-74.
Chaiphongpachara, T., S. Laojun and C. Kunphichayadecha, 2018. Effect of the CDC light trap on control of nocturnal mosquitoes in coastal Samut Songkhram Province, Thailand. Biodiversitas J. Bio. Divers., 19: 1750-1754.

Dujardin, J.P., 2008. Morphometrics applied to medical entomology. Infect. Genet. Evol., 8: 875-890.

Jirakanjanakit, N., S. Leemingsawat, S. Thongrungkiat, C. Apiwathnasorn and S. Singhaniyom et al., 2017. Influence of larval density or food variation on the geometry of the wing of Aedes (Stegomyia) aegypti. Trop. Med. Intl. Health, 12: 1354-1360.

Kaba, D., D. Berte, B.T.D. Ta, J. Telleria and P. Solano et al., 2017. The wing venation patterns to identify single tsetse flies. Infect. Genet. Evol., 47: 132-139.

Lorenz, C., F. Almeida, F. Almeida-Lopes, C. Louise and S.N. Pereira et al., 2017. Geometric morphometrics in mosquitoes: What has been measured?. Infect. Genet. Evol., 54: 205-215.

Rattanarithikul, R., E. Konishi and K.J. Linthicum, 1996. Detection of Plasmodium vivax and Plasmodium falciparum circumsporozoite antigen in anopheline mosquitoes collected in Southern Thailand. Am. J. Trop. Med. Hyg., 54: 114-121.

Rattanarithikul, R., R.E. Harbach, B.A. Harrison, P. Panthusiri and R.E. Coleman et al., 2010. Illustrated keys to the mosquitoes of Thailand. VI. Tribe Aedini. Southeast Asian J. Trop. Med. Public Health, 41: $1-38$.

Rohlf, F.J., 1999. Shape statistics: Procrustes superimpositions and tangent spaces. J. Classification, 16: 197-223.

Service, M., 2008. Medical Entomology for Students. 4th Edn., Cambridge University Press, Cambridge, UK. ISBN:978-0-521-70928-6, Pages: 299.

Suesdek, L., 2018. Microevolution of medically important mosquitoes-A review. Acta Trop., 19: 162-171.

Sumruayphol, S., C. Apiwathnasorn, N. Komalamisra, J. Ruangsittichai and Y. Samung et al., 2010. Bionomic status of Anopheles epiroticus Linton and Harbach, a coastal malaria vector, in Rayong Province, Thailand. Southeast Asian J. Trop. Med. Pub. Health, 41: 541-547.

Tainchum, K., M. Kongmee, S. Manguin, M.J. Bangs and T. Chareonviriyaphap, 2015. Anopheles species diversity and distribution of the Malaria vectors of Thailand. Trends Parasitol., 31: 109-119. 
WHO., 2007. Anopheline Species Complexes in South and South-East Asia Anopheline Species Complexes in South and South-East Asia. World Health Organization, Geneva, Switzerland, ISBN: 978-92-9022-294-1, Pages: 102.

WHO., 2013. Malaria Entomology and Vector Control. World Health Organization, Geneva, Switzerland, ISBN:9789241505802, Pages: 192.
WHO., 2017. World Malaria Report. World Health Organization, Geneva, Switzerland, ISBN: 9789241565523, Pages: 196.

Wilke, A.B.B., R.D.O. Christe, L.C. Multini, P.O. Vidal and R. Wilk-da-Silva et al., 2016. Morphometric wing characters as a tool for mosquito identification. PloS One, 11: 1-12. 